\title{
Skin Microbiota and its Interplay with Wound Healing
}

\author{
Marjana Tomic-Canic ${ }^{1} \cdot$ Jamie L. Burgess ${ }^{1} \cdot$ Katelyn E. O'Neill ${ }^{2} \cdot$ Natasa Strbo $^{2} \cdot$ Irena Pastar $^{1}$ (D)
}

Published online: 10 September 2020

(c) The Author(s) 2020

\begin{abstract}
The skin microbiota is intimately coupled with cutaneous health and disease. Interactions between commensal microbiota and the multiple cell types involved in cutaneous wound healing regulate the immune response and promote barrier restoration. This dialog between host cells and the microbiome is dysregulated in chronic wounds. In this review, we first describe how advances in sequencing approaches and analysis have been used to study the chronic wound microbiota, and how these findings underscored the complexity of microbial communities and their association with clinical outcomes in patients with chronic wound disorders. We also discuss the mechanistic insights gathered from multiple animal models of polymicrobial wound infections. In addition to the well-described role of bacteria residing in polymicrobial biofilms, we also discuss the role of the intracellular bacterial niche in wound healing. We describe how, in contrast to pathogenic species capable of subverting skin immunity, commensals are essential for the regulation of the cutaneous immune system and provide protection from intracellular pathogens through modulation of the antimicrobial molecule, Perforin-2. Despite recent advances, more research is needed to shed light on host-microbiome crosstalk in both healing and nonhealing chronic wounds to appropriately guide therapeutic developments.
\end{abstract}

\section{Key Points}

A complex microbiome is a hallmark of chronic nonhealing wounds.

Pathogenic bacteria are able to escape skin immunity and even reside inside host cells.

Commensal bacteria can modulate the cutaneous immune response to prevent wound infections.

Irena Pastar

IPastar@med.miami.edu

1 Dr. Phillip Frost Department of Dermatology and Cutaneous Surgery, Wound Healing and Regenerative Medicine Research Program, University of Miami, Miller School of Medicine, 1600 NW 10th Ave RMSB R-6056, Miami, FL 33136, USA

2 Department of Microbiology and Immunology, University of Miami Miller School of Medicine, Miami, FL, USA

\section{Introduction}

Chronic wounds, the most common of which are diabetic foot ulcers, pressure ulcers, venous leg ulcers, and nonhealing surgical wounds, are a major healthcare problem. Chronic wounds usually occur in older individuals with underlying conditions such as diabetes mellitus, vascular disease, and obesity [1]. Compromised immune and nutritional status, and chronic mechanical stress have also been shown to contribute to poor wound healing $[2,3]$. Chronic wounds are associated with alarmingly high mortality: the 5 -year mortality rates of ischemic (55\%), neuropathic (45\%), and neuroischemic (18\%) diabetic foot ulcers [4] are higher than or similar to those associated with breast cancer and prostate cancer (18\% and 8\%, respectively) [5]. Chronic wounds are also associated with high healthcare costs: in the USA, total spending estimates for chronic nonhealing wounds ranged from US\$28.1 to US\$96.8 billion in 2014 according to a retrospective analysis of the Medicare 5\% Limited Data Set [6]. Despite the alarming prevalence and high costs of care, efficient treatments are still lacking [2].

Cutaneous wound healing is a complex and very organized process, tightly controlled by several cell types through the secretion of growth factors, cytokines, and chemokines as illustrated in Fig. 1 [2]. Disruption of this process 


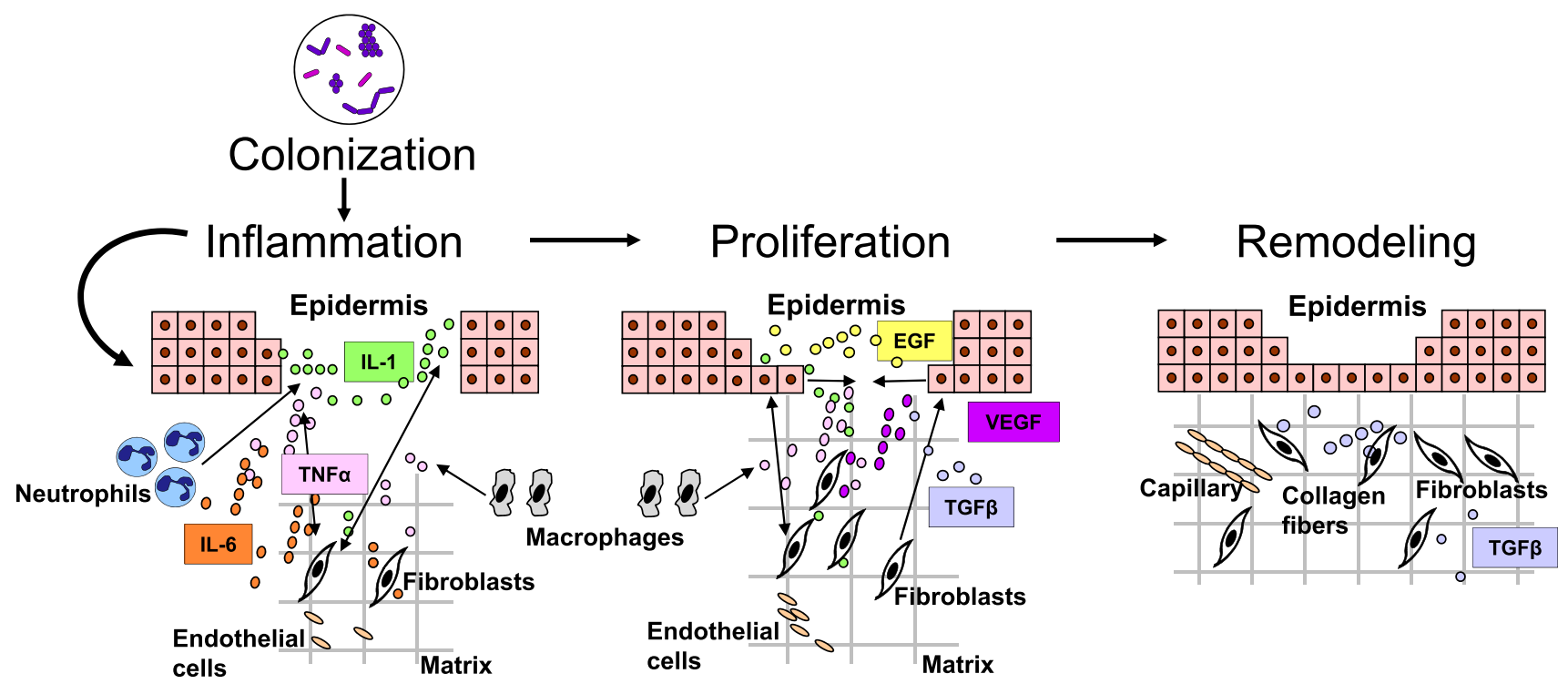

Fig. 1 Cutaneous wound healing progression: overlapping phases of acute healing. Epidermal keratinocytes, neutrophils, and macrophages are major cell types involved in the inflammatory response that occurs concurrently with hemostasis in the early stages of wound healing. Colonization of wounds with commensal microbiota may promote wound healing through activation of the innate immune response. In the proliferation phase, keratinocytes multiply

prevents the skin barrier from being properly restored; thus, the wound does not heal and becomes chronic. Higher rates of keratinocyte proliferation, an absence of migration, and fibrosis have all been observed in chronic wounds, regardless of their type. In contrast to the normal process of wound healing, angiogenesis, stem cell recruitment and activation, and extracellular matrix remodeling have all been shown to be impaired in chronic wounds, whereas inflammation has been found to be persistent and unresolved [2, 7-11]. Although the pathology of wound healing is now well characterized, the cellular and molecular mechanisms of impaired wound healing are still being studied. In particular, the role of microorganisms in chronic wound pathology is not fully understood and the importance of topical antimicrobial agents in their treatment is continuously debated.

Wounds provide an opportunity for microorganisms from the skin surface that constitute the skin microbiota, coupled with those from the environment, to gain entry to the underlying tissues and find optimal conditions for colonization and growth $[12,13]$. Interaction of commensal microorganisms with the skin cells during the normal cutaneous wound healing process is thought to be beneficial in modulating the innate immune response $[12,14,15]$. Conversely, pathogenic microorganisms are suspected to play a substantial role in delayed wound healing $[2,16]$. Thus, analyzing skin microbiota composition in both the normal acute and impaired wound healing processes is indispensable for the and migrate, fibroblasts migrate and deposit extracellular matrix, and angiogenesis occurs. Extracellular matrix remodeling results in scar formation and the ultimate restoration of the skin barrier. The most common growth factors and cytokines are shown. EGF epidermal growth factor, $I L$ interleukin, $T G F \beta$ transforming growth factor-beta, $T N F \alpha$ tumor necrosis factor-alpha, $V E G F$ vascular endothelial growth factor

identification of novel therapeutic strategies for patients with chronic wounds $[2,17]$.

In this review, we first discuss the undoubted benefits of the advances that have enabled analysis of the skin microbiota and highlight the challenges of applying such research to the field of wound healing. After describing the results obtained so far and presenting a forthcoming clinical application in patients with chronic wounds, we then provide updated information on the mechanisms by which the bacteria identified in these studies may contribute to delayed wound healing.

\subsection{Skin Microbiota Analysis in Chronic Wounds: Advances and Challenges}

Culture-based techniques have been used since the late 1800 s as the method of choice to identify bacteria constituting the skin microbiota. Although these techniques were useful, they only allowed identification of microorganisms that grew under usual laboratory conditions, which were not necessarily the most prominent microorganisms [17]. Moreover, the culture of anaerobic bacteria is fastidious and usually challenging $[17,18]$. Biases associated with culture-based approaches appear to have been overcome with the recent development of next-generation sequencing targeting the species-specific small subunit ribosomal RNA (16S rRNA) gene, a method that is now widely accessible $[17,18]$. This 
culture-independent molecular technique allows characterization of the wound microbiota according to all three of the dimensions considered as essential for understanding its role in wound healing: microbial load, microbial diversity, and the presence of pathogens [17]. The 16S rRNA sequencing method has shown high sensitivity in wound microbiota analyses, revealing greater bacterial diversity and load than traditional culture-based methods [17-19]. In a study of the microbiome of diabetic foot ulcers, culture-based techniques underestimated bacterial load by $2.34 \operatorname{logs}$ on average and by $>6 \log$ in some cases, and also failed to identify an average of at least 26 bacterial species per diabetic foot ulcer when compared with 16S rRNA sequencing [18]. Likewise, in another study of chronic wounds of various etiologies, 17 different bacterial taxa were identified with aerobic cultures, whereas 338 different bacterial taxa were identified in the same samples with 16S rRNA sequencing [19]. Of note, nine of the 20 most prominent bacteria identified with 16S rRNA sequencing in this study were anaerobes and would therefore not have been identified if aerobic cultures had been the only identification method used [19].

Although 16S rRNA sequencing addresses many of the limitations of culture-based approaches, it has its own limitations. Indeed, many bacteria can be identified using molecular-based techniques, but the active contribution of these bacteria to wound outcomes is unknown [17, 18, 20]. Furthermore, although sequencing may allow differences between "healing-capable" and "healing-impaired" individuals to be identified, the issue of correlation vs causation still remains. Gathering longitudinal data across multiple timepoints or assessing skin microbiota composition before disease manifestation or at the initial onset of a wound may help to find causative links [21]. Moreover, complementary approaches, including metagenomic analyses, should be incorporated into studies of wound healing because 16S rRNA sequencing cannot identify nonbacterial microorganisms (e.g., fungi and viruses), which also constitute the skin microbiota and may influence wound healing outcomes [17, 18, 22]. The metagenomic approach (shotgun DNA sequencing) not only allows profiling of all microbial genomes in a sample, it also allows functional and metabolic pathway analyses based on the sequences of DNA fragments throughout the genomes. Unlike $16 \mathrm{~S}$ rRNA sequencing, the metagenomic approach provides identification of microbiome compositions at the strain-level resolution [21].

Beyond the methods used to investigate skin microbiota composition, further factors should be considered to achieve high-quality, standardized skin microbiome research and enable reproducibility and comparisons between studies. Two recent studies $[21,23]$ identified potential pitfalls related to skin microbiome research, and established guidelines for conducting and reporting such investigations that encouraged consideration of the study design, skin sample collection/storage, sample processing, sequencing approaches, required controls, analysis pipelines, and data deposition.

\section{Microbiota Composition of Chronic Wounds}

\subsection{Individuals at Risk of Chronic Wound Development}

Diabetic foot ulcers, a common complication of diabetes, are a widespread type of chronic wound $[2,24]$. To investigate whether changes in composition were present in the skin microbiome of individuals at risk of developing these lesions, Redel et al. [25] conducted a case-control observational study using high-throughput 16S rRNA sequencing technology to analyze the skin microbiota from the arms and feet of diabetic men without any previous history of diabetic foot ulcers and from those of matched nondiabetic men as controls. Although microbiota composition and total bacterial counts were similar in the arm samples of both groups, higher bacterial diversity was observed in the plantar foot samples of diabetic men compared to nondiabetic men. The relative abundance of Firmicutes was lower in diabetic men after adjustment for the false discovery rate, whereas the abundance of Actinobacteria was found to be higher. Moreover, the plantar foot samples of diabetic men displayed a lower relative abundance of Staphylococcus species overall than those of nondiabetic men. However, a higher quantity of more virulent forms of Staphylococcus aureus characterized the foot microbiota in diabetic patients [25]. These skin microbiota differences between diabetic and nondiabetic individuals may be linked to the risk of future diabetic foot ulcer development [25]. Similar studies are needed to investigate potential changes in the cutaneous microbiome in at risk populations, e.g., patients with venous insufficiency at risk of venous leg ulcer development and patients with spinal cord injury at risk of pressure ulcer development.

\subsection{Individuals with Chronic Wounds}

Initial studies examining microbiota in chronic wounds using 16S rRNA sequencing did not investigate correlations between microbiota composition and wound outcomes. Based on a review of five microbiome studies, Misic et al. [17] found most bacteria colonizing chronic wound tissue belonged to 21 families, among which Staphylococcaceae and Pseudomonadaceae were predominant regardless of the etiology of the wound and the type of sampling [18, 26-29]. Moreover, a large-scale retrospective study [20] of the microbiota from 2963 samples from chronic wounds of various etiologies showed that Staphylococcus and Pseudomonas 
were the most common genera, identified in $63 \%$ and $25 \%$ of the wounds, respectively. Staphylococcus aureus and $S$. epidermidis were the predominant species, and methicillinresistant Staphylococcus species were identified in about 25\% of the chronic wounds [20]. Of note, commensal bacteria including Corynebacterium and Propionibacterium species, as well as anaerobic bacteria, were highly prevalent in the analyzed chronic wound samples [20]; however, the microbiota of chronic wounds is distinct from the surrounding healthy skin microbiota. No significant differences in microbiota composition were observed across wound types [20]. Additional smaller scale studies also identified S. aureus as the most common species in chronic wounds of various etiologies [19] and in neuropathic diabetic foot ulcers [18, 24]. In the study by Gardner et al. [18], associations between microbiota composition and clinical factors were identified: high microbial diversity, as well as increases in the relative abundance of anaerobic bacteria and Gram-negative Proteobacteria, were observed in deep ulcers and in those of long duration, whereas a great abundance of Staphylococcus, mainly $S$. aureus, was found in superficial ulcers and in those of short duration. The same group evaluated the temporal dynamics of the microbiota colonizing diabetic foot ulcers and found that rapid and dynamic changes in the microbiota were associated with faster healing and better outcomes [24]. Using shotgun metagenomics, Kalan et al. [16] identified strain-specific differences in the microbiome of diabetic foot ulcers that correlated with clinical outcomes of healing. Two clinical $S$. aureus isolates were found to be associated with nonhealing wounds, SA10757 and SA7372, and healing inhibition was confirmed in mouse models. In contrast, wounds infected with Alcaligenes faecalis, a common environmental bacterium, healed at rates similar to controls [16]. Further investigation revealed that $A$. faecalis was associated with high levels of interleukin- 8 and other cytokines that enhance wound healing [16]. These studies indicate that functional level differences between microbiota species, or even between specific microbiota strains, may play an important role in determining the clinical outcomes of chronic wounds.

\section{Structural Organization of the Skin Microbiota in Chronic Wounds: The Biofilm}

\subsection{Biofilm Description and Characteristics in Wounds}

Microorganisms constituting the chronic wound microbiota have been shown to be mainly organized in biofilms, i.e., a complex microbial community containing bacteria and fungi surrounded by a polymeric matrix composed of polysaccharides, lipids, proteins, and nucleic acids [30, 31]. Using light and scanning electron microscopy techniques, James et al.
[32] identified biofilms in $60 \%$ of chronic wounds but in only $6 \%$ of acute wounds. In a systematic review and metaanalysis gathering data from eight prospective cohort studies including 185 chronic wounds and one case report series [33], the prevalence of biofilms in nonhealing human chronic wounds assessed by microcopy-associated in some cases with molecular methods-was $78.2 \%$, varying between 60 and $100 \%$. Therefore, biofilms are suspected to contribute to chronic wound pathology and impaired wound healing.

\subsection{Can Biofilms in Chronic Wounds be Used for Diagnostic Purposes?}

Because of the predominance of biofilms in chronic wounds and the role of bacteria able to form biofilms in impaired wound healing (see Sect. 4.1), a device or a method that could quickly detect the presence of biofilms in a wound would be useful for developing effective treatment options and for monitoring treatment progress [31]. Schultz and Sampson [34] described one method for detecting biofilms based on staining with ruthenium red or Alcian blue to reveal the mucopolysaccharides of the biofilm matrix [34]. In a prospective observational study testing the clinical applicability of this noninvasive method, Nakagami et al. [35] found that it could be used to predict changes in wound slough (biofilm) development in patients with pressure ulcers who had undergone sharp wound debridement and wound washing before biofilm detection. In a more recent retrospective observational study [36], the same group demonstrated that this biofilm detection method could be used to monitor the effect of biofilm elimination by sharp debridement: 1 week after blotting, the percentage decrease in wound area was significantly higher in the biofilm-eliminated group than in the biofilm-remaining group, and the presence of remaining biofilms was an independent predictive factor for lower percentage decreases in the wound area [36]. These results showed that biofilm removal using sharp debridement improved pressure ulcer healing. This quick and noninvasive method could therefore be useful for helping clinicians eliminate entire biofilms by detecting and mapping their distribution on the wound. A larger clinical trial is needed to validate this method for clinical use.

\section{Mechanisms by Which the Skin Microbiota Contribute to Delayed Wound Healing: Findings from Experimental Models}

\subsection{Extracellular Bacteria Found in Biofilms}

Biofilms can develop from a single bacterial or fungal species, can be polymicrobial (i.e., consist of several species), or can even span kingdoms by being composed of interacting bacteria and fungi [31]. Several studies have reported 
that most chronic nonhealing wounds are polymicrobial in nature, including a large-scale retrospective analysis conducted by Wolcott et al. [20] in which 93\% $(n=2963)$ of samples from wounds of various etiologies were polymicrobial. Thus, investigating crosstalk among species is essential for understanding the regulation of wound healing. With this aim, our group used a well-established porcine wound model to study the interactions between two of the most prevalent bacterial species identified in both acute and chronic wounds of various etiologies: S. aureus and Pseudomonas aeruginosa [13]. Using methicillin-resistant $S$. aureus (MRSA) USA300-0114 and a P. aeruginosa clinical wound isolate, we showed that both species co-existed in porcine cutaneous wounds in vivo [13]. We also found that MRSA did not affect the growth of $P$. aeruginosa, but that $P$. aeruginosa reduced MRSA growth in the mixed-species biofilms both in vivo and in vitro, with a lower effect observed in vivo than in vitro [13]. Moreover, the presence of P. aeruginosa in the co-infected wounds led to higher expression of two MRSA virulence factors: Panton-Valentine leukocidin (pvl) at 2 and 4 days post-wounding, and $\alpha$-hemolysin (Hla) at 4 days post-wounding [13]. Finally, we showed that in comparison with uninfected wounds and wounds infected with single-species biofilms, wounds co-infected with $P$. aeruginos $a$ and MRSA showed delayed epithelialization through down-regulation of keratinocyte growth factor 1 expression [13]. Mainly produced by fibroblasts during the acute wound healing process, keratinocyte growth factor 1 is known to play an important role in re-epithelialization by stimulating keratinocyte proliferation and migration in a paracrine manner [13]. In conclusion, we observed that interactions within mixed-species biofilms resulted in a decreased load but increased virulence of MRSA, and significantly delayed wound healing in comparison with uninfected wounds and wounds infected with single-species biofilms [13]. Similar findings from murine $[37,38]$ and rabbit [39] wound infection models underscore the contribution of polymicrobial wound environments to impaired host responses and inhibition of healing. Future studies utilizing metatranscriptomics (RNA transcript sequencing) with the ability to identify the full range of actively transcribed genes from both the host and the microbiome will enable in-depth analyses of host-microbe interactions in the wound environment.

\subsection{Intracellular Bacterial Niche}

Although biofilms play an important role in the recurrent infections observed in patients with chronic wounds, other factors are involved in chronic wound infections. We hypothesized that, in addition to extracellular bacteria, the intracellular microbial niche contributes to the impairment of wound healing. Keratinocytes constitute the first cellular barrier to infection at the skin level. Like innate immune cells, they were recently shown to constitutively express Perforin-2 (P-2) in both mice and humans [40]. Perforin-2 is an ancient innate immune protein that is highly conserved from sponges to humans [40, 41]. Perforin-2 is located within the membranes of endosomal vesicles, which traffic to and fuse with bacteria-containing phagosomes thus enabling elimination of intracellular bacteria [40, 42, 43]. Perforin- 2 has been shown to be critical for the clearance of a variety of Gram-positive and Gram-negative pathogens, highlighting its pivotal role in the host's innate antimicrobial response [17].

To determine the bactericidal role of $\mathrm{P}-2$ against MRSA in vivo, our group studied the effect of infecting P-2 deficient mice, heterozygous mice, and wild-type mice with a traditionally sub-lethal load of MRSA after tape stripping [17]. Twelve days after MRSA epicutaneous infection, MRSA could only be recovered from the internal organs of a few heterozygous mice, whereas wild-type mice only had recoverable colony-forming units in the skin. However, bacterial colony-forming units were recovered from the blood, spleen, and kidney of all P-2-deficient mice. These animals failed to eliminate the MRSA and eventually succumbed to infection. We have also investigated P-2 expression and cellular distribution in human skin [44]. Using an amplified fluorescence in situ hybridization coupled with cell sorting (FISH-Flow) technique, we found that P-2 transcripts were detected in gamma delta $T$ cells, endothelial cells, keratinocytes, and fibroblasts, confirming P-2 expression by both professional and nonprofessional immune cells (Fig. 2). In addition, we showed that a keratinocyte cell line overexpressing a P-2GFP fusion protein cleared intracellular $S$. aureus infection more efficiently than control cells, supporting our findings of the importance of P-2 in bacterial clearance in the skin [44]. We also used an ex vivo human wound model to evaluate $\mathrm{P}-2$ expression during wound healing and to determine how levels change in response to the most common skin and wound pathogen, S. aureus. We found that although P-2 expression was induced during wound healing, S. aureus infection inhibited P-2 expression in both hematopoietic and nonhematopoietic cells resulting in inhibition of wound closure (Fig. 2) [44].

Conversely, skin commensals, including S. epidermidis, have been found to promote healing and to induce expression of different antimicrobials [14]. Given the prevalence of $S$. aureus in chronic wounds, it seems likely that $S$. aureus suppression of P-2 promotes bacterial persistence at the wound site resulting in wound chronicity. Conversely, the presence of commensal bacteria may modify the skin and wound environment to prevent or resolve bacterial wound infections. Further studies on the mechanisms of P-2 action have the potential to provide major advances in our understanding of innate immunity in the skin, and the insights gained could be applied to diseases that compromise the skin barrier without causing chronic wounds, such as atopic dermatitis. 
a

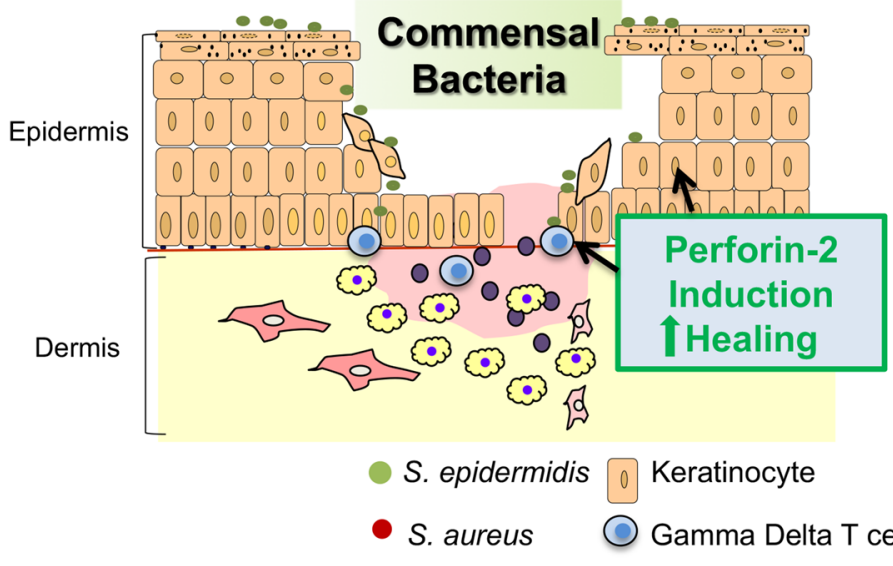

b

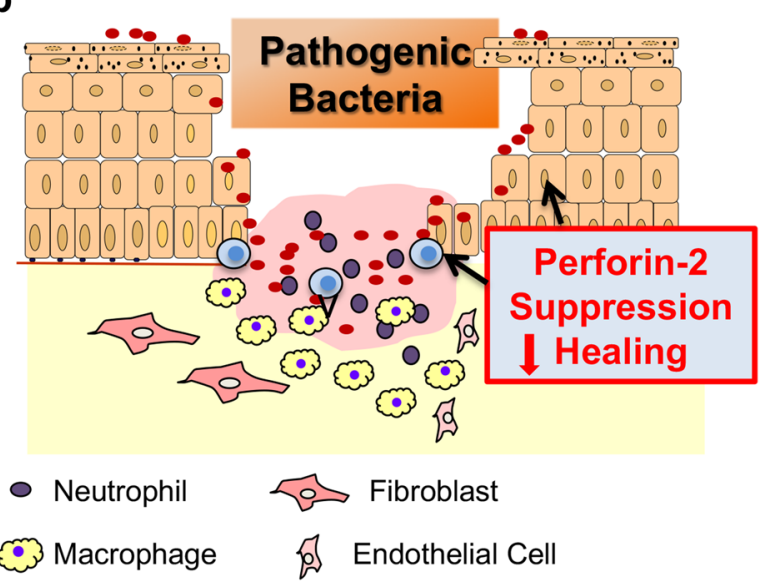

Fig. 2 Cutaneous immunity is differentially regulated by commensal and pathogenic microorganisms through modulation of Perforin-2. a Colonization of the wound with commensal bacteria may promote wound healing by inducing antimicrobial proteins such as Perforin-2,

\section{Conclusions and Perspectives}

Recent advances have enabled better characterization of bacteria in chronic wounds. More importantly, the latest studies did not only describe "what is there?", but also established correlations with wound healing outcomes and provided insights on "what is it doing?". This is the area of active investigation that continuously reveals new potential pathways for therapeutic intervention for patients with chronic wounds. Microorganisms constituting "the chronic wound microbiota" are mainly organized in the form of a polymicrobial biofilm. Interactions between different species within the polymicrobial environment have been shown to be dynamic and to modify bacterial behavior, resulting in increased virulence and delayed wound healing. It is necessary to further investigate such microbiomehost interactions to identify new potential treatments. Indeed, our own research showed that antibiotic-resistant bacteria can subvert P-2 to prevent them from being killed. Understanding how one of the most frequent wound colonizers, S. aureus, evades the antimicrobial activity of P-2, as well as how commensal bacteria may prevent or reverse the inhibitory effect of pathogens, will provide fundamental new insights into the intricate mechanisms by which commensal and pathogenic bacteria mediate crosstalk with the cutaneous immune system during wound healing. Understanding the mechanisms of P-2 activation will enable the development of therapies to clear antibiotic-resistant chronic wound pathogens. It would also be interesting to assess whether molecular analyses of chronic wound microbiota could be used to determine treatment efficacy and enhance the development of new therapies. Because of the complexity and dynamics of the cutaneous wound healing process, the development of combination treatments-targeting both the host and the microbiome-could be necessary thus stimulating a protective immune response against pathogenic bacteria. b Wound infection with pathogenic bacteria results in Perforin-2 suppression in both hematopoietic and nonhematopoietic cells and inhibition of healing

to augment healing and prevent and treat infections in wounds, skin, and soft tissues.

Acknowledgements The authors thank Laurence Rous, PhD, Emma Pilling, PhD, and Marielle Romet, PhD (Synergy Pharm-Santé Active Edition) for medical writing assistance funded by Laboratoires Dermatologiques Avène, Pierre Fabre Dermo-Cosmétique.

\section{Declarations}

Funding Medical writing assistance was funded by Laboratoires Dermatologiques Avène, Pierre Fabre Dermo-Cosmétique. The work presented was in part funded by the National Institutes of Health, National Institute for Nursing Research (NR015649).

Conflict of interest Marjana Tomic-Canic, Jamie L. Burgess, Katelyn E. O'Neill, Natasa Strbo, and Irena Pastar have no conflicts of interest that are directly relevant to the content of this article.

Disclosure This article is published as part of a journal supplement wholly funded by Laboratoires Dermatologiques Avène, Pierre Fabre Dermo-Cosmétique.

Author Contributions MTC, NS, and IP: contributed to the conception of the manuscript; IP, MTC, JLB, KO, and NS: performed the literature search and data analysis, and critically revised the work. All authors edited the final draft.

Open Access This article is licensed under a Creative Commons Attribution-NonCommercial 4.0 International License, which permits any non-commercial use, sharing, adaptation, distribution and reproduction in any medium or format, as long as you give appropriate credit to the original author(s) and the source, provide a link to the Creative Commons licence, and indicate if changes were made. The images or other third party material in this article are included in the article's Creative Commons licence, unless indicated otherwise in a credit line to the material. If material is not included in the article's Creative Commons licence and your intended use is not permitted by statutory 
regulation or exceeds the permitted use, you will need to obtain permission directly from the copyright holder. To view a copy of this licence, visit http://creativecommons.org/licenses/by-nc/4.0/.

\section{References}

1. Gould L, Abadir P, Brem H, Carter M, Conner-Kerr T, Davidson $\mathrm{J}$, et al. Chronic wound repair and healing in older adults: current status and future research. Wound Repair Regen. 2015;23(1):1-13.

2. Eming SA, Martin P, Tomic-Canic M. Wound repair and regeneration: mechanisms, signaling, and translation. Sci Transl Med. 2014;6(265):265sr6

3. Fayne RA, Borda LJ, Egger AN, Tomic-Canic M. The potential impact of social genomics on wound healing. Adv Wound Care (New Rochelle). 2020;9(6):325-31.

4. Moulik PK, Mtonga R, Gill GV. Amputation and mortality in newonset diabetic foot ulcers stratified by etiology. Diabetes Care. 2003;26(2):491-4.

5. Armstrong DG, Wrobel J, Robbins JM. Guest editorial: are diabetes-related wounds and amputations worse than cancer? Int Wound J. 2007;4(4):286-7.

6. Nussbaum SR, Carter MJ, Fife CE, DaVanzo J, Haught R, Nusgart $\mathrm{M}$, et al. An economic evaluation of the impact, cost, and Medicare policy implications of chronic nonhealing wounds. Value Health. 2018;21(1):27-32.

7. Ramirez HA, Pastar I, Jozic I, Stojadinovic O, Stone RC, Ojeh N, et al. Staphylococcus aureus triggers induction of miR-15B-5P to diminish DNA repair and deregulate inflammatory response in diabetic foot ulcers. J Invest Dermatol. 2018;138(5):1187-96.

8. Stone RC, Stojadinovic O, Rosa AM, Ramirez HA, Badiavas E, Blumenberg $\mathrm{M}$, et al. A bioengineered living cell construct activates an acute wound healing response in venous leg ulcers. Sci Transl Med. 2017;9(371):eaaf8611.

9. Stone RC, Stojadinovic O, Sawaya AP, Glinos GD, Lindley LE, Pastar I, et al. A bioengineered living cell construct activates metallothionein/zinc/MMP8 and inhibits TGFbeta to stimulate remodeling of fibrotic venous leg ulcers. Wound Repair Regen. 2020;28(2):164-76.

10. Pastar I, Wong LL, Egger AN, Tomic-Canic M. Descriptive vs mechanistic scientific approach to study wound healing and its inhibition: is there a value of translational research involving human subjects? Exp Dermatol. 2018;27(5):551-62.

11. Thom SR, Hampton M, Troiano MA, Mirza Z, Malay DS, Shannon S, et al. Measurements of CD34+/CD45-dim stem cells predict healing of diabetic neuropathic wounds. Diabetes. 2016;65(2):486-97.

12. Zeeuwen PL, Boekhorst J, van den Bogaard EH, de Koning HD, van de Kerkhof PM, Saulnier DM, et al. Microbiome dynamics of human epidermis following skin barrier disruption. Genome Biol. 2012;13(11):R101.

13. Pastar I, Nusbaum AG, Gil J, Patel SB, Chen J, Valdes J, et al. Interactions of methicillin resistant Staphylococcus aureus USA300 and Pseudomonas aeruginosa in polymicrobial wound infection. PLoS One. 2013;8(2):e56846.

14. Harrison OJ, Linehan JL, Shih HY, Bouladoux N, Han SJ, Smelkinson $\mathrm{M}$, et al. Commensal-specific $\mathrm{T}$ cell plasticity promotes rapid tissue adaptation to injury. Science. 2019;363(6422):eaat6280.

15. Lai Y, Di Nardo A, Nakatsuji T, Leichtle A, Yang Y, Cogen AL, et al. Commensal bacteria regulate Toll-like receptor 3-dependent inflammation after skin injury. Nat Med. 2009;15(12):1377-82.

16. Kalan LR, Meisel JS, Loesche MA, Horwinski J, Soaita I, Chen $\mathrm{X}$, et al. Strain- and species-level variation in the microbiome of diabetic wounds is associated with clinical outcomes and therapeutic efficacy. Cell Host Microbe. 2019;25(5):641-55.e5.

17. Misic AM, Gardner SE, Grice EA. The wound microbiome: modern approaches to examining the role of microorganisms in impaired chronic wound healing. Adv Wound Care (New Rochelle). 2014;3(7):502-10.

18. Gardner SE, Hillis SL, Heilmann K, Segre JA, Grice EA. The neuropathic diabetic foot ulcer microbiome is associated with clinical factors. Diabetes. 2013;62(3):923-30.

19. Rhoads DD, Wolcott RD, Sun Y, Dowd SE. Comparison of culture and molecular identification of bacteria in chronic wounds. Int J Mol Sci. 2012;13(3):2535-50.

20. Wolcott RD, Hanson JD, Rees EJ, Koenig LD, Phillips CD, Wolcott RA, et al. Analysis of the chronic wound microbiota of 2963 patients by $16 \mathrm{~S}$ rDNA pyrosequencing. Wound Repair Regen. 2016;24(1):163-74.

21. Kong HH, Andersson B, Clavel T, Common JE, Jackson SA, Olson ND, et al. Performing skin microbiome research: a method to the madness. J Invest Dermatol. 2017;137(3):561-8.

22. Kalan L, Loesche M, Hodkinson BP, Heilmann K, Ruthel G, Gardner SE, et al. Redefining the chronic-wound microbiome: fungal communities are prevalent, dynamic, and associated with delayed healing. mBio. 2016;7(5):e01058-116.

23. Grogan MD, Bartow-McKenney C, Flowers L, Knight SAB, Uberoi A, Grice EA. Research techniques made simple: profiling the skin microbiota. J Invest Dermatol. 2019;139(4):747-52. e1.

24. Loesche M, Gardner SE, Kalan L, Horwinski J, Zheng Q, Hodkinson BP, et al. Temporal stability in chronic wound microbiota is associated with poor healing. J Invest Dermatol. 2017;137(1):237-44.

25. Redel H, Gao Z, Li H, Alekseyenko AV, Zhou Y, PerezPerez GI, et al. Quantitation and composition of cutaneous microbiota in diabetic and nondiabetic men. J Infect Dis. 2013;207(7):1105-14.

26. Price LB, Liu CM, Frankel YM, Melendez JH, Aziz M, Buchhagen $\mathbf{J}$, et al. Macroscale spatial variation in chronic wound microbiota: a cross-sectional study. Wound Repair Regen. 2011;19(1):80-8

27. Price LB, Liu CM, Melendez JH, Frankel YM, Engelthaler D, Aziz M, et al. Community analysis of chronic wound bacteria using 16S rRNA gene-based pyrosequencing: impact of diabetes and antibiotics on chronic wound microbiota. PLoS One. 2009;4(7):e6462.

28. Wolcott RD, Gontcharova V, Sun Y, Dowd SE. Evaluation of the bacterial diversity among and within individual venous leg ulcers using bacterial tag-encoded FLX and titanium amplicon pyrosequencing and metagenomic approaches. BMC Microbiol. 2009;27(9):226.

29. Frank DN, Wysocki A, Specht-Glick DD, Rooney A, Feldman RA, St Amand AL, et al. Microbial diversity in chronic open wounds. Wound Repair Regen. 2009;17(2):163-72.

30. Monroe D. Looking for chinks in the armor of bacterial biofilms. PLoS Biol. 2007;5(11):e307.

31. Phillips PL, Wolcott RD, Fletcher J, Schultz GS. Biofilms made easy. Wounds Int. 2010;1(3):1-6.

32. James GA, Swogger E, Wolcott R, Pulcini E, Secor P, Sestrich $\mathrm{J}$, et al. Biofilms in chronic wounds. Wound Repair Regen. 2008;16(1):37-44.

33. Malone M, Bjarnsholt T, McBain AJ, James GA, Stoodley P, Leaper $\mathrm{D}$, et al. The prevalence of biofilms in chronic wounds: a systematic review and meta-analysis of published data. J Wound Care. 2017;26(1):20-5.

34. Schultz GS, Phillips PL, Sampson EM. Materials and methods for assessing and mapping microbes and microbial biofilms 
on wound. US Patent Application Publication, Pub No.: US 2012/0322048 A1; 2012.

35. Nakagami G, Schultz G, Gibson DJ, Phillips P, Kitamura A, Minematsu T, et al. Biofilm detection by wound blotting can predict slough development in pressure ulcers: a prospective observational study. Wound Repair Regen. 2017;25(1):131-8.

36. Nakagami G, Schultz G, Kitamura A, Minematsu T, Akamata K, Suga $\mathrm{H}$, et al. Rapid detection of biofilm by wound blotting following sharp debridement of chronic pressure ulcers predicts wound healing: a preliminary study. Int Wound J. 2020;17(1):191-6.

37. Dalton T, Dowd SE, Wolcott RD, Sun Y, Watters C, Griswold JA, et al. An in vivo polymicrobial biofilm wound infection model to study interspecies interactions. PLoS One. 2011;6(11):e27317.

38. Dhall S, Do D, Garcia M, Wijesinghe DS, Brandon A, Kim J, et al. A novel model of chronic wounds: importance of redox imbalance and biofilm-forming bacteria for establishment of chronicity. PLoS One. 2014;9(10):e109848.

39. Seth AK, Geringer MR, Hong SJ, Leung KP, Galiano RD, Mustoe TA. Comparative analysis of single-species and polybacterial wound biofilms using a quantitative, in vivo, rabbit ear model. PLoS One. 2012;7(8):e42897.
40. McCormack RM, de Armas LR, Shiratsuchi M, Fiorentino DG, Olsson ML, Lichtenheld MG, et al. Perforin-2 is essential for intracellular defense of parenchymal cells and phagocytes against pathogenic bacteria. Elife. 2015;24(4):e06508.

41. McCormack R, Podack ER. Perforin-2/Mpeg1 and other pore-forming proteins throughout evolution. J Leukoc Biol. 2015;98(5):761-8.

42. Pang SS, Bayly-Jones C, Radjainia M, Spicer BA, Law RHP, Hodel AW, et al. The cryo-EM structure of the acid activatable pore-forming immune effector macrophage-expressed gene 1 . Nat Commun. 2019;10(1):4288.

43. Ni T, Jiao F, Yu X, Aden S, Ginger L, Williams SI, et al. Structure and mechanism of bactericidal mammalian perforin-2, an ancient agent of innate immunity. Sci Adv. 2020;6(5):eaax8286.

44. Strbo N, Pastar I, Romero L, Chen V, Vujanac M, Sawaya AP, et al. Single cell analyses reveal specific distribution of anti-bacterial molecule Perforin-2 in human skin and its modulation by wounding and Staphylococcus aureus infection. Exp Dermatol. 2019;28(3):225-32. 